\title{
Deformation Mechanics of a Non-linear Hyper-viscoelastic Porous Material, Part I: Testing and Constitutive Modeling of Non-porous Polychloroprene
}

\author{
Christopher Salisbury $^{1} \cdot$ Duane Cronin $^{1} \cdot$ Fue-Sang Lien ${ }^{1}$
}

Received: 20 May 2015/Accepted: 11 July 2015 / Published online: 21 July 2015

(C) Society for Experimental Mechanics, Inc 2015

\begin{abstract}
Solid and foamed polymeric materials demonstrate a significant increase in stiffness with increasing deformation rate, and existing hyper-viscoelastic constitutive formulations are often limited in applicability across large ranges of deformation rate and finite deformations. The development of micro-level pore-based foam models requires the mechanical properties of the constituent nonporous material coupled with efficient and representative constitutive models. In this study, the mechanical properties of non-porous polychloroprene were measured at low deformation rates using a conventional hydraulic test apparatus and at high deformation rates using a polymeric split Hopkinson pressure bar apparatus. A constitutive model was developed using an additive formulation to describe the hyper-viscoelastic material response for large deformations and a range of deformation rates from quasistatic $\left(0.001 \mathrm{~s}^{-1}\right)$ up to $2700 \mathrm{~s}^{-1}$. The material coefficients were determined using a constrained optimization technique that simultaneously fit all of the data, and iterated to determine the required number of material constants. The constitutive model was implemented into an explicit finite element code and accurately predicted the response of nonporous polychloroprene rubber $\left(R^{2}=0.996\right)$ over the range of tested strain rates. Importantly, the finite element implementation minimized the required computational storage, addressing limitations in existing constitutive models, and was computationally efficient, which was necessary for the large finite element micro-scale
\end{abstract}

Duane Cronin

duane.cronin@uwaterloo.ca

1 Department of Mechanical and Mechatronics Engineering, University of Waterloo, 200 University Ave. West, Waterloo, ON, Canada simulations of foamed polychloroprene undertaken in a follow-on study.

Keywords Mechanical properties - Polychloroprene rubber · Hyper-viscoelastic · Non-linear constitutive model $\cdot$ Large deformation $\cdot$ High strain rate $\cdot$ Split Hopkinson pressure bar

\section{Introduction}

Many materials demonstrate nonlinear response over large deformations and a significant increase in stiffness with increasing deformation rate, which are important considerations for analysis and design in energy absorption applications and impact problems [1]. Measuring the mechanical properties of low impedance materials such as polymers at high deformation rates is therefore important [2], and must be supported by representative constitutive models for use in advanced modeling techniques such as the finite element method to evaluate materials and designs. Although mechanical testing techniques exist for soft or low impedance materials, the accurate measurement of mechanical properties requires appropriate sample geometry and test methods $[3,4]$. The mechanical response of a polymer to an applied load is often described in terms of the elastic (instantaneous) and time dependent (viscoelastic) response [5-7]. Ultimately, the stress at any point in a material can be a function of strain, strain rate and strain history (path dependent materials). For small deformations and a modest range of strain rates, linear treatments of the elastic and viscoelastic response are often sufficient to describe material response. Larger deformations have commonly been addressed through the use of hyperelastic descriptions [8, 9] coupled to linear 
viscoelastic formulations using a superposition approach (quasi-linear viscoelasticity or QLV) [10]; however, when the deformations are large $(>50 \%)$ and the strain rates span multiple decades (less than $0.1 \mathrm{~s}^{-1}$ to greater than $1000 \mathrm{~s}^{-1}$ ) these models are not sufficient to describe the significant nonlinearities in the material response. Large deformation and a wide range of deformation rates are often encountered in impact scenarios where materials may be used for energy absorption and this is an important consideration in representing materials in models to support analysis and design.

The motivation for the current study was to characterize non-porous polychloroprene rubber so that these properties could be used to predict the behavior of porous, foamed polychloroprene at the micro-scale as part of a larger study on the deformation mechanics of hyperelastic porous media. This aspect is further discussed in Part II of this study. To address this complex topic, a constitutive model was required to address large deformations over a wide range of strain rates, followed by implementation of this model in a finite element code capable of incorporating fluid/structure analysis to include the effect of nitrogen in the foamed material. It was determined that existing viscoelastic and quasi-linear viscoelastic models could not accurately represent the material response over the measured range of deformation and deformation rates. Implementation of a constitutive model in a finite element code is often the ultimate goal of model development, but is often not undertaken due to the significant level of effort since many models are not developed for this purpose. Therefore, the objectives of Part I of this study were to: measure the mechanical properties of non-porous polychloroprene rubber across a range of deformation rates and large deformations, develop a descriptive constitutive model, determine the constitutive model parameters, and validate the constitutive model for use in an explicit finite element model.

\section{Background}

\section{Experimental Testing}

In impact scenarios involving large deformations it is particularly important to characterize a material over a representative range of strains, which can be as high as 80-95\% engineering strain $[3,11,12]$, to ensure the model is predictive. The measurement of mechanical properties for low impedance materials presents several challenges including representative sample size, achievement of dynamic equilibrium (uniform deformation) within the specimen, and minimizing radial inertia effects [3]. For this study, low (up to $0.1 \mathrm{~s}^{-1}$ ) and intermediate $\left(\sim 10 \mathrm{~s}^{-1}\right)$ deformation rate testing was undertaken on a hydraulic test apparatus, while high deformation rate testing $\left(\sim 2700 \mathrm{~s}^{-1}\right)$ was undertaken using a polymeric split Hopkinson pressure bar (PSHPB) apparatus, which is often used for compression testing of low impedance materials [3, 11-14]. The apparatus comprises three $25.4 \mathrm{~mm}$ diameter acrylic bars. The incident and transmitter bars were $2.4 \mathrm{~m}$ in length with $1000 \Omega$ semi-conductor strain gauges [3] located at the bar mid-points. The striker bar was $710 \mathrm{~mm}$ in length, to avoid wave superposition effects in the incident and transmitted bar signals. Although polymeric bars are known to result in wave attenuation and dispersion [12, 15], this can be readily addressed using viscoelastic wave propagation methods. In this study, the experimental method of Bacon [16] was used with a onedimensional wave propagation analysis [3, 14] to determine a set of wave propagation coefficients. Although other methods have been used to address the challenges of testing low impedance materials with metallic bars including pulse shaping [17] and load cells mounted at the bar ends the current study used a PSHPB apparatus. The primary benefits of the polymeric bars include a longer load application or rise time $(\sim 115 \mu$ s compared to $\sim 10 \mu$ s for metallic bars) to allow for equilibrium of the sample and achieve uniform deformation, an improved impedance match with the test sample compared to metallic bars resulting in an improved measured signal to noise ratio, and a large striker bar length allowing for large deformations to be attained.

\section{Hyper-viscoelastic Constitutive Model}

Viscoelastic or deformation rate effects are often modeled through the superposition of the viscoelastic stress contribution $\left(\sigma_{i j}^{v}\right)$ to the hyperelastic Cauchy stress $\left(\sigma_{i j}^{e}\right)($ Eq. 1) and this has been implemented in many finite element codes including explicit time domain (e.g. LS-Dyna [6]) and implicit finite element codes (e.g. Abaqus [7]).

$\sigma_{i j}=\sigma_{i j}^{e}+\sigma_{i j}^{v}$

The hyperelastic (nonlinear elastic) response can be described by many different forms [18] such as the Ogden model (Eq. 2).

$\tau_{i i}^{e}=J \sigma_{i i}^{e}=\lambda_{i} \frac{\partial W}{\partial \lambda_{i}}=\sum_{k=1}^{N_{k}} \mu_{k}\left(\tilde{\lambda}_{i}^{\alpha_{k}}-\frac{1}{3} a_{k}\right)+p$

where:

$a_{k}=\tilde{\lambda}_{1}^{\alpha_{k}}+\tilde{\lambda}_{2}^{\alpha_{k}}+\tilde{\lambda}_{3}^{\alpha_{k}}$.

In the previous equation, $\lambda$ is the stretch, $\mu$ and $\alpha$ are material constants that number from 1 to $N_{k}$ and $p$ is an arbitrary volumetric pressure term. The number of material 
constants, $N_{k}$, are determined by the algorithm used to fit the material model to the experimental data and are usually limited to three. Including viscoelastic or strain history effects in numerical codes can be challenging due to the strain path dependency of the material and was primarily enabled by the development of the convolution integral (Appendix 1, [19]) (Eq. 4). The approach used for implementation in an explicit or time-domain finite element code, is described as:

$\sigma_{i j}^{v}=\sum_{k=1}^{N_{k}} 2 \gamma_{k} \int_{0}^{t} e^{-\beta_{k}(t-\phi)} \frac{d \varepsilon_{i j}^{d e v}}{d \phi} d t$

where the deviatoric strains are given by:

$\varepsilon_{i j}^{d e v}=\varepsilon_{i j}-\frac{1}{3} \varepsilon_{i j} \delta_{i j}$.

The numerical implementation of Eqs. 4 and 5 use a Prony series approximation for the relaxation function, where $\gamma$ is a constant as described in Appendix 1.

The primary issue with these models is the inability to predict the strain rate sensitivity of rubber-like materials through the convolution integral alone. As discussed by Malvern [20], the convolution integral provides a "fading memory" which acts over a period of time similar to that of a stress relaxation or creep test. In this study, the Ogden model was used to describe the hyperelastic component. Although this model is often presented with an additive viscoelastic Prony Series as described in Appendix 1, initial investigations determined that this method could not adequately model the material response over the large range of strain rates considered. Yang et al. [5] developed a model to account for higher loading rates based on the summation of a hyperelastic Mooney-Rivlin model [9] and a modifier on the convolution integral. The form of the viscoelastic contribution was defined as:

$\sigma^{v}=-p^{v}+\mathbf{F}(t) \cdot \stackrel{t}{\Omega}\{\mathbf{C}(\phi)\} \mathbf{F}^{T}(t)$

where $\Omega$ is a functional describing how the strain history acts upon the stress, and $p^{v}$ is an arbitrary pressure. They assumed this functional to be of the form

$\stackrel{t}{\Omega}\{\mathbf{C}(\phi)\}=\int_{-\infty}^{t} \Phi\left(I_{1}, I_{2}\right) m(t-\phi) \dot{\mathbf{E}} d \phi$

where the strain rate, $\dot{\mathbf{E}}$, is given by

$\dot{\mathbf{E}}=\frac{1}{2}\left(\dot{\mathbf{F}}^{T} \cdot \mathbf{F}+\mathbf{F}^{T} \cdot \dot{\mathbf{F}}\right)$.

The function, $\Phi$, was assumed to be:

$\Phi=A_{4}+A_{5}\left(I_{2}-3\right)$

where $I_{2}$ is the second invariant of stretch and $A_{4}, A_{5}$ are material constants. The function $m$ is defined as a Prony series with one coefficient. Through the combination of the Mooney-Rivlin hyperelastic model and the viscoelastic addition, Yang et al. were able to obtain reasonable fits to data at high rates of strain for two similar rubbers. However, the implementation of the model in Yang et al. was limited by their choice of a Prony series with only one coefficient. Additionally, their model required storage of the entire deformation gradient (9 variables) as well as the six stress components ( 15 memory locations for each element), which is computationally prohibitive for large finite element models. For example, the 570,000 element model presented in the second part of this study requires 8.6 million storage locations, compared to 3.4 million for the present model, which must be accessed for each computational cycle.

The constitutive model that was developed for the current study sought to address the limitations seen by the relative insensitivity of the linear viscoelastic constitutive models to deformation rates that are commonly available in commercial finite element codes, as well as the storage limitation in the model proposed by Yang et al.

\section{Methods}

\section{Mechanical Testing of Polychloroprene}

Mechanical testing was undertaken on samples of a polychloroprene rubber, a cross-linked elastomer (Rubatex LLC), over a wide range of strain rates $\left(0.001-2700 \mathrm{~s}^{-1}\right)$. Test samples were manufactured using a special cutting technique, required to achieve consistent samples due to the flaccid nature of the material. A custom sharpened coring tool $(10 \mathrm{~mm}$ in diameter, Fig. 1a) in a milling machine was used to cut cylindrical samples from the original sheet material at a feed rate of $3.75 \mathrm{~mm} / \mathrm{s}$ with the tool spinning at $1500 \mathrm{rpm}$. Distilled water was used as a lubricant in order to reduce friction between the tool and material, which could otherwise lead to non-uniform sample geometry. The specimens were then cut to the desired length $(4 \mathrm{~mm})$ by placing them into a custom fixture (Fig. 1b). The hole in the fixture was slightly smaller than that of the sample creating a small interface force to hold the sample in place while cutting with a sharp utility knife. Again, distilled water was used as a lubricant to minimize friction and generate samples with parallel faces.

Mechanical testing was undertaken to measure the compressive mechanical properties using two different test apparatus to obtain the desired strain rates. A quasi-static testing apparatus (Fig. 2) comprising a standard hydraulic test frame with a Model 407 controller (MTS, MN, USA) was used in displacement control mode to obtain high resolution data in the lower strain rate regime of 
Fig. 1 a Coring tool for cylindrical samples, b sample length cutting fixture
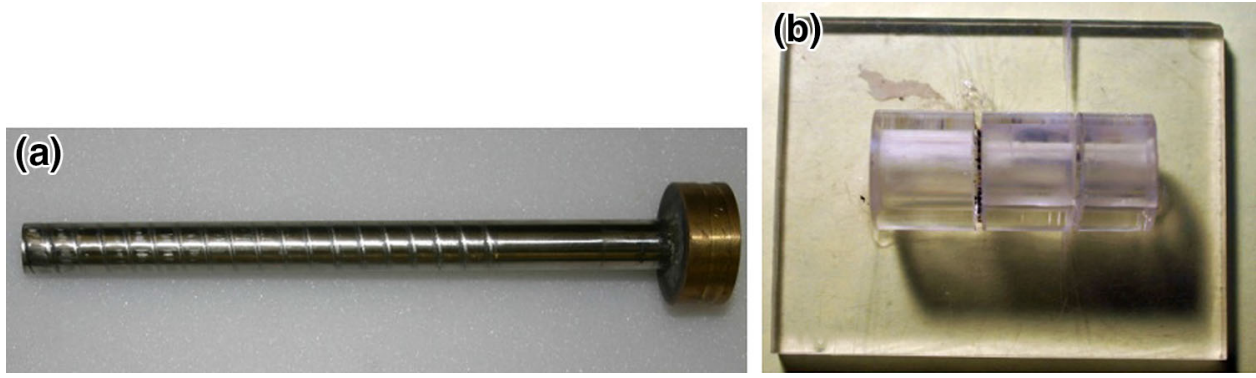

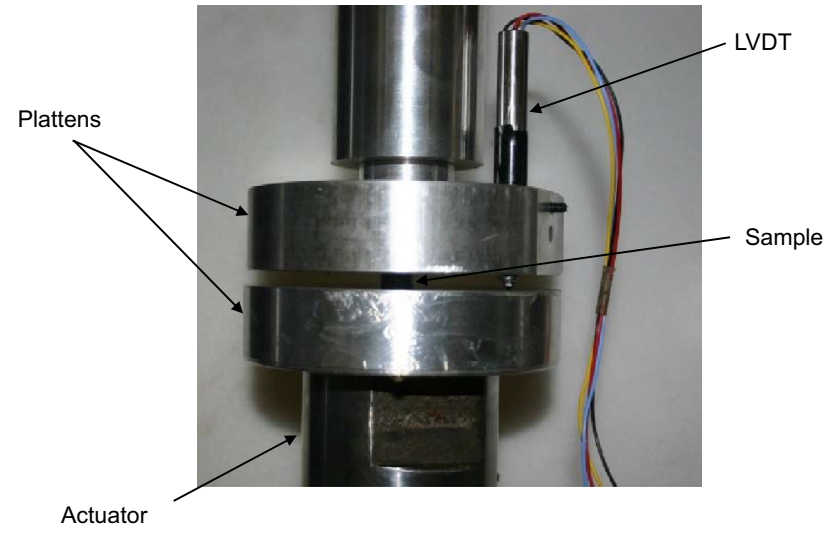

Fig. 2 Quasi-static compressive test apparatus with sample

$0.001-10 \mathrm{~s}^{-1}$. Load was measured with a $2225 \mathrm{~N}$ (500 lbf) load cell (Strain Sert, model FL05U(C)-2SP); necessary to provide appropriate measurement resolution for this low stiffness material, and displacement was accurately measured using a linear variable differential transformer (LVDT) (Omega, type LD-320-7.5) which was capable of measuring engineering strains up to $80 \%$. The specimen was compressed between two parallel aluminum platens that were lapped after machining to ensure a smooth surface and lightly lubricated with lithium-based grease. The time that the specimen was in contact with the grease was minimized to prevent any effect on the material, but was necessary to prevent barreling or non-uniform deformation of the specimen. Three test samples at each strain rate were found to be sufficient since the material demonstrated relatively low variability.

The dynamic experiments were performed using a PSHPB apparatus $[3,14]$. The bars were made from acrylic (PMMA) to provide good impedance match with the polychloroprene rubber. The ends of the bars were lubricated with a thin layer of high pressure lithium grease, which was necessary to minimize frictional effects and prevent barreling of the sample during the test. Tests were conducted within $1 \mathrm{~min}$ of placing the sample in contact with the lubricant. A custom fixture was used to align the sample with the center of the bars to minimize any off-axis loading. From previous testing on RTV rubbers [21] and ballistic gelatin
[3], it was determined that a specimen length of $4 \mathrm{~mm}$ was suitable for the characterization of this material. In order to prevent any preloading of the samples, the gap between the bars was set to the measured gauge length of the sample using precisely machined slip gauges. This allowed the bars to contact the sample without preloading it.

Dynamic equilibrium in the samples was evaluated using the measured forces at end of the incident and transmitted bars determined from the incident, transmitted and reflected strain waves (Fig. 3). The waves were propagated to the bar ends using experimentally determined wave propagation coefficients $[3,14]$ and the bar end forces were calculated from these waveforms (Fig. 4). The forces at the bar ends coincide well during the loading section of the curve from 0 to $0.0012 \mathrm{~s}$ up to the peak load in the test. It can be seen that the forces begin to diverge later in time, particularly after $0.0014 \mathrm{~s}$; however, this occurs during the unloading phase and is beyond the region of interest in the test (e.g. following the peak load). A minimum of three samples were tested at each strain rate and the results were averaged for the data analysis, described in detail below. It should be noted that the quoted strain rates are nominal values since the strain rate did vary over the duration of the test; however, the actual strain rate history was used when computing the specimen response and fitting the material models developed in this study.

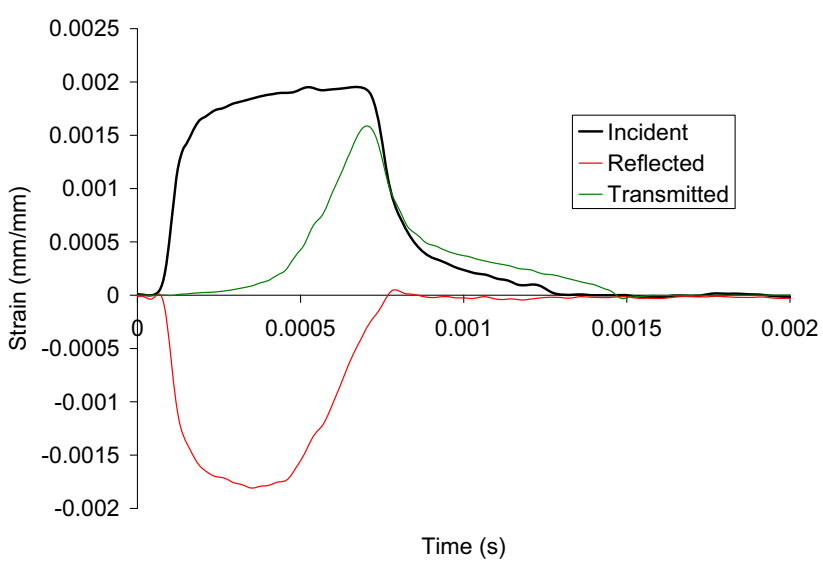

Fig. 3 Typical incident, reflected and transmitted waves for a dynamic test $\left(2700 \mathrm{~s}^{-1}\right)$ 


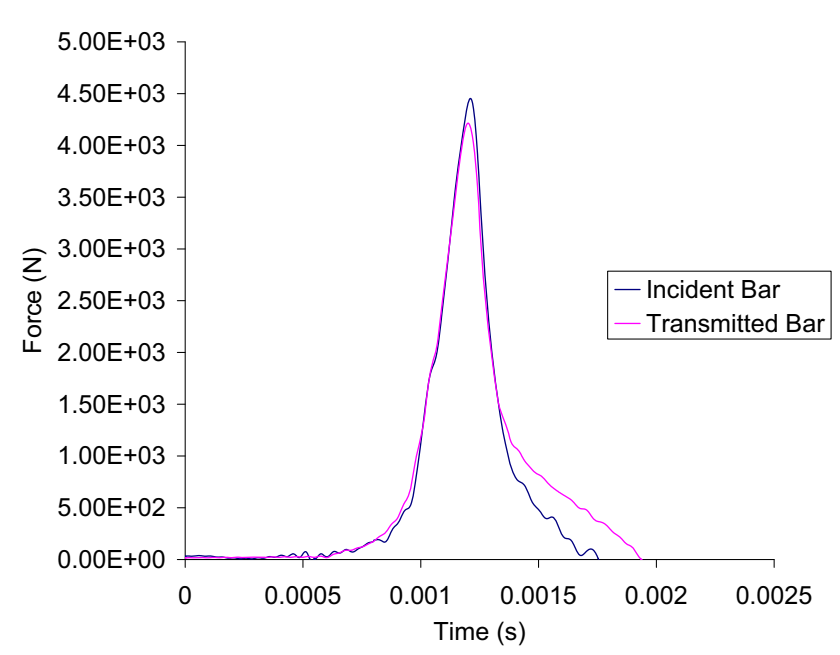

Fig. 4 Force-time curves for a dynamic test demonstrating equilibrium

\section{Hyper-viscoelastic Constitutive Model Development}

The constitutive models described previously were investigated with the measured mechanical data, but were limited in their ability to model the entire strain and strain rate range considered. The primary limitation was the inability for QLV to match the material response across the wide range of strain rates in the measured experimental data, and the large memory requirements for other nonlinear methods. To address these limitations, an additive approach was investigated, combining the Ogden hyperelastic constitutive model and incorporating viscoelasticity through the convolution integral, combined with a Rivlin-type series modifier to account for non-linear effects (Eq. 10) based on the invariants of the stretch tensor on a principal basis. The selection of this approach was based on investigation of the available models for the experimental data, and considering several different possible forms for the constitutive model, not presented here for brevity.

$\sum_{i=0, j=0, k=0}^{\infty} A_{i j k}\left(I_{1}-3\right)^{i}\left(I_{2}-3\right)^{j}\left(I_{3}-3\right)^{k}, A_{000}=0$

Rewriting Eq. 22 (Appendix 1) in terms of the principal viscoelastic stresses, $\tau_{i i}^{v}$ (here the Kirchhoff stress is used instead of the Cauchy stress where $\tau=J \sigma$ ), principal stretches, $\lambda_{i}$, and including the modifier terms gives:

$\tau_{i i}^{v}(t)=\int_{-\infty}^{t} \Gamma G(t-\phi) \frac{d \lambda_{i}(t)}{d \phi} d \phi+p^{v}$ (no summation)

where $p^{v}$ is the viscoelastic contribution to the arbitrary pressure and the modifier, $\Gamma$, is given as:
$\Gamma \equiv \sum A_{p q r}\left(I_{1}-3\right)^{p}\left(I_{2}-3\right)^{q}\left(I_{3}-3\right)^{r}$

Here $p, q$ and $r$ each range from 0 to the number of terms required in the fit to the material data. The third invariant, $I_{3}$, is approximately equal to 1 for an incompressible material and so the last term in Eq. 12 was not included. Therefore, Eq. 12 can be expressed as

$\Gamma \equiv \sum A_{p q}\left(I_{1}-3\right)^{p}\left(I_{2}-3\right)^{q}$

which, when expanded, gives:

$$
\begin{aligned}
\Gamma= & \sum A_{p q}\left(I_{1}-3\right)^{p}\left(I_{2}-3\right)^{q} \\
= & A_{00}+A_{01}\left(I_{2}-3\right)+A_{02}\left(I_{2}-3\right)^{2}+A_{10}\left(I_{1}-3\right) \\
& +A_{11}\left(I_{1}-3\right)\left(I_{2}-3\right)+\cdots
\end{aligned}
$$

Following the evolution of the time marching technique to solve the convolution integral (described in Appendix 1) the increment in viscoelastic stress (Eq. 32, Appendix 1), can be written as:

$$
\begin{aligned}
\int_{t}^{t+\Delta t} \Gamma G(t+\Delta t-\phi) \frac{d \lambda_{i}(\phi)}{d \phi} d \phi \\
\quad=\sum_{k=1}^{N_{k}} \Gamma \frac{\gamma_{k}}{\beta_{k}} \frac{\lambda_{i}(t+\Delta t)-\lambda_{i}(t)}{\Delta t}\left[1-e^{-\beta_{k} \Delta t}\right]
\end{aligned}
$$

The history variable (Eq. 36, Appendix 1), was then given as:

$\sum_{k=1}^{N_{k}} H_{k}(t) \equiv \int_{0}^{t} \Gamma \sum_{k=1}^{N} \gamma_{k} e^{-\beta_{k}(t-\phi)} \frac{d \lambda_{i}(\phi)}{d \phi} d \phi$

As with Eq. 38 (Appendix 1), Eqs. 15 and 16 were combined to give

$$
\begin{aligned}
\tau_{i i}^{v}(t+\Delta t)= & \sum_{k=1}^{N_{k}}\left[e^{-\beta_{k} \Delta t} H_{k}(t)+\Gamma \frac{\gamma_{k}}{\beta_{k}} \frac{\lambda_{i}(t+\Delta t)-\lambda_{i}(t)}{\Delta t}\left[1-e^{-\beta_{k} \Delta t}\right]\right] \\
& +p^{v} \text { (no summation) }
\end{aligned}
$$

The total state of stress was then determined using Eq. 17 with Eq. 2 through the linear sum $\tau_{i i}^{T}=\tau_{i i}^{e}+\tau_{i i}^{\nu}$ (no summation). The total principal stresses were expressed as

$$
\begin{aligned}
\tau_{i i}^{T}(t+\Delta t)= & \tau_{i i}^{e}+\tau_{i i}^{v} \text { (no summation) } \\
= & \sum_{d=1}^{N_{d}} \mu_{d}\left(\tilde{\lambda}_{i}^{\alpha_{d}}-\frac{1}{3} a_{d}\right)+p \\
& +\sum_{k=1}^{N_{k}}\left[e^{-\beta_{k} \Delta t} H_{k}(t)+\Gamma \frac{\gamma_{k}}{\beta_{k}} \frac{\lambda_{i}(t+\Delta t)-\lambda_{i}(t)}{\Delta t}\left[1-e^{-\beta_{k} \Delta t}\right]\right] \\
& +p^{v}
\end{aligned}
$$

where $p$ and $p^{v}$ are scalars which can be summed as $p^{T}$ to give: 


$$
\begin{aligned}
\tau_{i i}^{T}(t+\Delta t)= & \sum_{d=1}^{N_{d}} \mu_{d}\left(\tilde{\lambda}_{i}^{\alpha_{d}}-\frac{1}{3} a_{d}\right) \\
& +\sum_{k=1}^{N_{k}} e^{-\beta_{k} \Delta t} H_{k}(t) \quad \text { (no summation) } \\
& +\Gamma \frac{\gamma_{k}}{\beta_{k}} \frac{\lambda_{i}(t+\Delta t)-\lambda_{i}(t)}{\Delta t}\left[1-e^{-\beta_{k} \Delta t}\right]+p^{T}
\end{aligned}
$$

The general solution procedure for Eq. 19 used a time marching technique as described in Appendix 2, which was beneficial for implementation into an explicit finite element program. A material subroutine was written, verified and used in the data analysis and curve fitting. It should be noted that after the total principal stresses are calculated using Eq. 19, the stresses are rotated back to the standard basis using $\tau_{i j}=\tau_{i} n_{i} n_{j}$ (summation in force). However, in the case of uniaxial tests the principal and standard bases are the same so rotation is not necessary in this specific case.

\section{Constitutive Model Parameter Estimation}

Non-linear models, such as that proposed in Eq. 19, require specific care and data processing to accurately estimate the material constants. Prior to determining the constants for the constitutive model, the experimental data was manipulated into a form that was compatible with the constitutive model. The deformation tensor $F_{i j}$ for the uniaxial case assuming incompressibility can be written as

$$
F_{i j}=\left[\begin{array}{ccc}
\lambda_{1} & 0 & 0 \\
0 & \lambda_{1}^{-1 / 2} & 0 \\
0 & 0 & \lambda_{1}^{-1 / 2}
\end{array}\right]
$$

Using Eqs. 17 and 19, and the fact that the stresses $\tau_{22}, \tau_{33}=0$ since the tests are uniaxial, the arbitrary pressure, $p^{T}$, can be calculated as follows: at each time step the principal stretches, $\lambda_{i}$, are determined from the deformation gradient, then the material constants $\mu, \alpha, \lambda$ and $\beta$ are known along with the history variable $H_{k}$ which is available from the previous time step. Since the total principal stresses $\tau_{22}, \tau_{33}$ are equal to zero, Eq. 19 can be rearranged to give:

$$
\begin{aligned}
p^{T}= & -\sum_{d=1}^{N_{d}} \mu_{d}\left(\tilde{\lambda}_{i}^{\alpha_{d}}-\frac{1}{3} a_{d}\right) \\
& -\sum_{k=1}^{N_{k}} e^{-\beta_{k} \Delta t} H_{k}(t)-\Gamma \frac{\gamma_{k}}{\beta_{k}} \frac{\lambda_{2}(t+\Delta t)-\lambda_{2}(t)}{\Delta t}\left[1-e^{-\beta_{k} \Delta t}\right] .
\end{aligned}
$$

If the arbitrary hyperelastic and viscoelastic pressure components are desired, they can be solved for individually using a similar analysis. It should be noted that this pressure is arbitrary and does not necessarily represent the actual pressure within the material due to the incompressibility assumption. Given that the bulk modulus of the material greatly exceeded the stresses created in the material during testing, neglecting compressibility was considered valid.

The data from each experimental test was resampled in such a manner to ensure that equal spacing between stretch points was achieved as well as equal numbers of points for each curve. The data was manipulated to this form to prevent biasing of the coefficients to one of the curves or one area of a particular curve (i.e. if one of the six curves used had 1000 points instead of 100, when the fitting of the material constants was performed, the coefficients would be biased towards the curve with 1000 points). Similarly, if one area of a curve had more points (for example 1000 points between stretches of 1 to 0.8 and 100 points from 0.79 to 0.2 ) the coefficients would be biased towards that area.

Several challenges were encountered when attempting to use conventional linear regression fitting procedures such as those found in commercial programs (e.g. [22]). The difficulty was in the determination of the convolution integral, which as discussed in the previous section, requires a time marching approach that does not lend itself to common approaches and so specialized programs are required for parameter fitting [23]. To address this requirement, a program was written in MATLAB [24] to calculate the material parameters using constrained optimization techniques. This technique was based on an approach where the difference between all of the experimental curves and predicted curves was minimized simultaneously, while ensuring that the coefficients of the material model remained positive, as required from the derivation. An iterative approach including changing the number of material constants was performed to optimize the number of parameters and their values for the constitutive model. A discussion of the optimization technique and the methodology for choosing the initial values for the material constants parameters can be found in [25].

\section{Results and Discussion}

\section{Mechanical Compression Test Results}

A minimum of three tests were performed at each strain rate and the data was resampled to give equal increments in strain so that an average curve for each strain rate could be created, since this was required for the constitutive model parameter estimation. The mechanical test data was found to be very consistent between samples at the same strain rate, with examples shown in Fig. $5\left(0.001 \mathrm{~s}^{-1}\right)$ and Fig. 6 
$\left(2700 \mathrm{~s}^{-1}\right)$. Only minor deviations from the average curve were noted at lower strain rates (0.001-7.9 $\left.\mathrm{s}^{-1}\right)$. The tests performed at $2700 \mathrm{~s}^{-1}$ (Fig. 6) demonstrated the largest spread between tests; however, the data was considered very consistent up to a compressive stretch of 0.4 .

The polychloroprene rubber investigated in this study demonstrated a dependence on strain rate over the entire loading history, as shown in the resulting average curves (Fig. 7). This is further highlighted (Fig. 8) when considering stress values at different values of stretch versus strain rate, plotted on a logarithmic scale. As the strain rate increased from 0.001 to $2700 \mathrm{~s}^{-1}$, the resulting stress values increased from -1.75 to $-15.5 \mathrm{MPa}$ at a stretch of 0.4. Similarly, at a stretch of 0.2 the stress increased from -8.75 to $-74.25 \mathrm{MPa}$ over the same increase in strain rate. Additionally, the polychloroprene rubber exhibited a nonlinear viscoelastic effect (Fig. 8) since the values of stress did not increase linearly with the logarithm of strain rate.

\section{Constitutive Model Parameter Determination and Comparison}

Table 1 shows the results for the maximized coefficient of determination or $R^{2}$ value for the experimental tests conducted. As indicated in the table, an excellent $R^{2}$ value of 0.9962 was achieved using the constitutive model. This result was achieved with one set of Ogden parameters, two nonlinear modifier terms and five sets of Prony series constants, where the $\beta$ parameters span six decades ranging from $10^{-7}$ to $10^{-1}$, corresponding to the strain rate range of the experimentally measured data. It should be noted that, commonly, one set of Prony series constants is used for each decade in time corresponding to the deformation rate. However, enforcing additional parameters did not improve the curve fit and was therefore the number of parameters optimized by the algorithm was used.

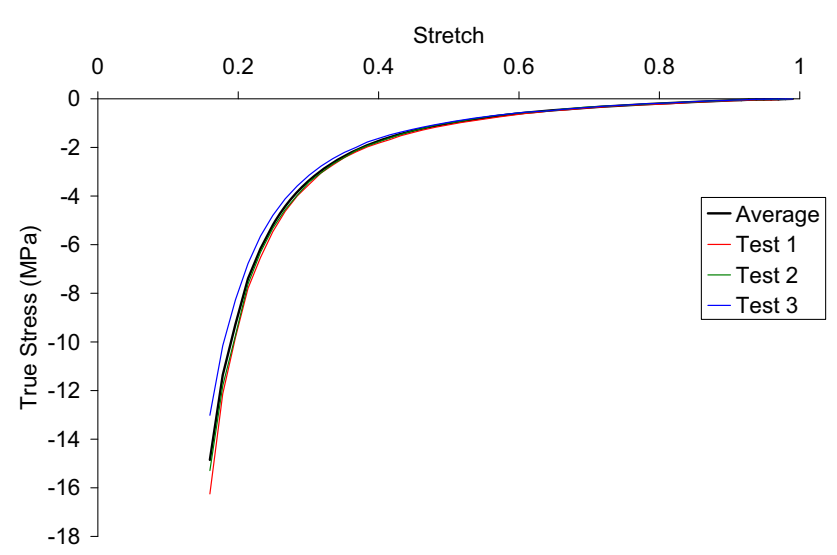

Fig. 5 Stress-stretch response for non-porous polychloroprene rubber $\left(0.001 \mathrm{~s}^{-1}\right)$

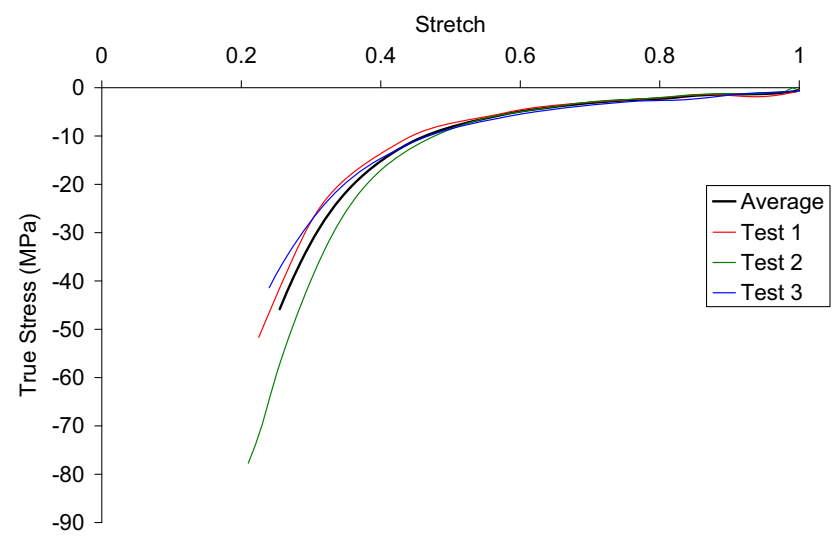

Fig. 6 Stress-stretch response for polychloroprene rubber $\left(2700 \mathrm{~s}^{-1}\right)$

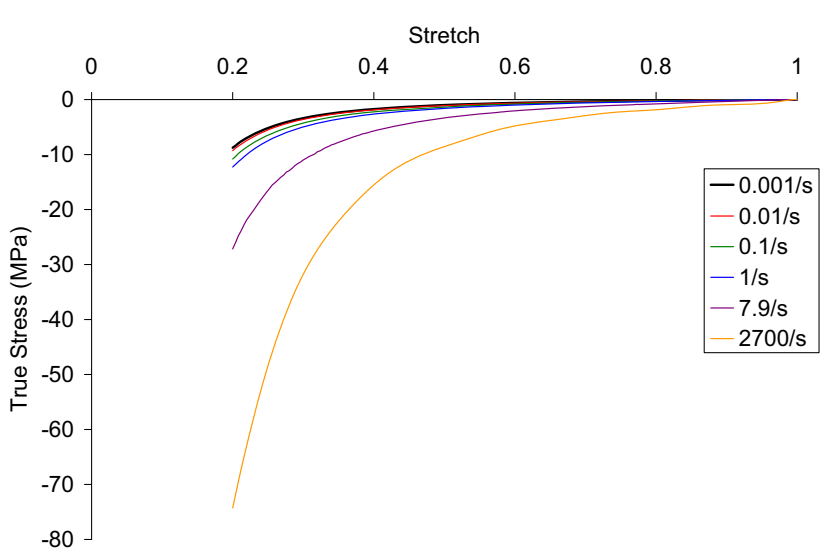

Fig. 7 Average stress-stretch curves for polychloroprene rubber over the strain rates tested

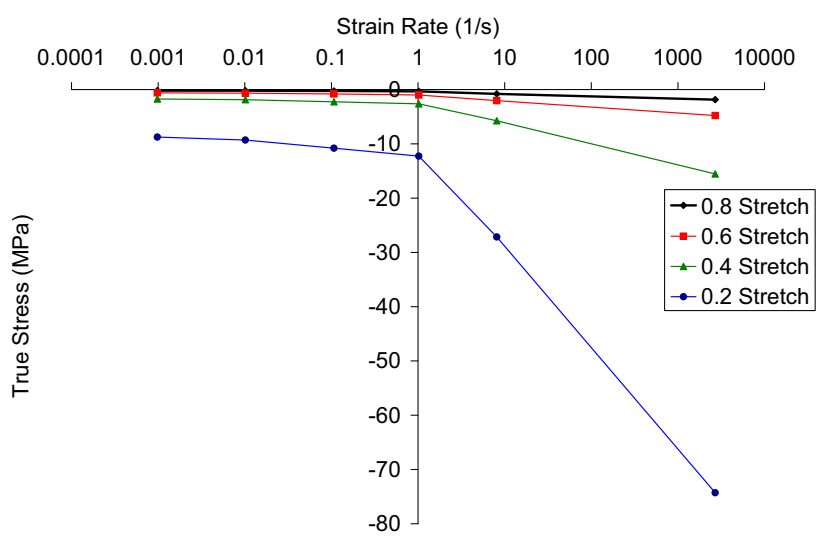

Fig. 8 Stresses at different stretch values over the range of strain rates tested

Figure 9 shows the experimental data and the results from the constitutive model for the six strain rates. In general there was a good correspondence between the constitutive model and the experimental data over the tested strain rates. At rates between 0.001 and $0.1 \mathrm{~s}^{-1}$ the 
Table 1 Constitutive model material parameters

\begin{tabular}{ll}
\hline $\begin{array}{l}\text { Best fit } \\
\text { Number of Ogden coefficient sets: } 1\end{array}$ & $\mathrm{R}^{2}=0.99615$ \\
\hline \multicolumn{2}{l}{ Number of Prony series coefficients sets: 5} \\
\hline Number of modifier terms: 2 & \\
\hline$\mu_{1}=199.7(\mathrm{kPa})$ & $\alpha_{1}=1.118(-)$ \\
$\gamma_{1}=43.61(\mathrm{kPa})$ & $\beta_{1}=5.26 \mathrm{E}-07\left(\mathrm{~ms}^{-1}\right)$ \\
$\gamma_{2}=6.381(\mathrm{kPa})$ & $\beta_{2}=2.38 \mathrm{E}-04\left(\mathrm{~ms}^{-1}\right)$ \\
$\gamma_{3}=88.08(\mathrm{kPa})$ & $\beta_{3}=0.141\left(\mathrm{~ms}^{-1}\right)$ \\
$\gamma_{4}=123.3(\mathrm{kPa})$ & $\beta_{4}=0.2478\left(\mathrm{~ms}^{-1}\right)$ \\
$\gamma_{5}=147.9(\mathrm{kPa})$ & $\beta_{5}=0.2181\left(\mathrm{~ms}^{-1}\right)$ \\
$\mathrm{A}_{00}=11.02(-)$ & \\
$\mathrm{A}_{01}=94.42(-)$ &
\end{tabular}

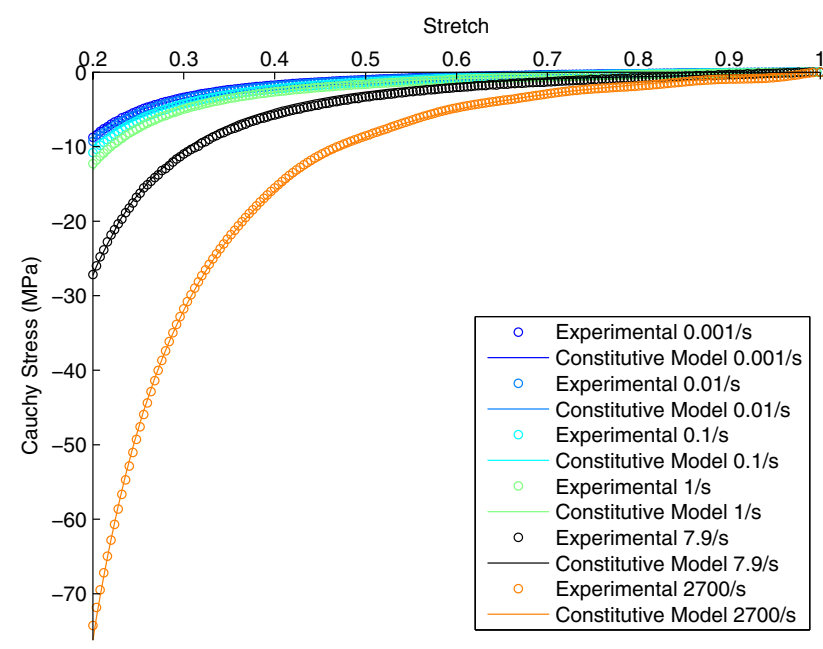

Fig. 9 Experimental data and constitutive model for the optimized $R^{2}$ case

data was well modeled with a slight under prediction of the stress at stretches of approximately 0.2 . Similarly, at $1 \mathrm{~s}^{-1}$ the stress was slightly over predicted at large deformations. For the $7.9 \mathrm{~s}^{-1}$ case the stress was slightly under predicted at stretches between 0.65 and 0.2. There was an excellent correspondence at $2700 \mathrm{~s}^{-1}$ with only very minor deviations of the model from the data. The $R^{2}$ value for each strain rate range was calculated to further illustrate the excellent correspondence between the experimental and constitutive model. As indicated in Table 2, the relative $\mathrm{R}^{2}$ values ranged from 0.9937 to 0.9996 for the 7.9 and $2700 \mathrm{~s}^{-1}$ cases respectively. The average of the $\mathrm{R}^{2}$ values was 0.9962 (Table 2). As a test of the optimization solver, the analysis was rerun with the same initial guesses as recommended in Ref. [26]. Additionally, the optimization analysis was rerun with the constants in Table 1 as the initial guess. In both cases, the same set of parameters and $R^{2}$ value was identified by the algorithm.
Table 2 Individual $\mathrm{R}^{2}$ comparison of experimental curves to constitutive model

\begin{tabular}{ll}
\hline Strain rate $\left(\mathrm{s}^{-1}\right)$ & $\mathrm{R}^{2}(-)$ \\
\hline 0.001 & 0.99698 \\
0.01 & 0.99707 \\
0.1 & 0.99580 \\
1 & 0.99373 \\
7.9 & 0.99370 \\
2700 & 0.99962 \\
Average & 0.99615 \\
\hline
\end{tabular}

\section{Numerical Implementation of the Constitutive Model}

A user material model subroutine was written to incorporate the constitutive model into a commercial non-linear explicit finite element program (LS-Dyna, LSTC [6]). To verify the material model, single element simulations were conducted and the output compared to the results of the constitutive model and experiments using a cubic single solid element model with unity dimensions. Displacement constraints were applied to the element as shown in Fig. 10 where the red arrows indicate that the nodes had fixed coordinates and the experimental displacement history was applied to the nodes indicated by the open black arrows in the $\mathrm{x}$-direction. The experimental displacement history was scaled to account for the unity element dimensions so that the strain rate was maintained for the six single element simulations. The stress and stretch for the unit element was recorded for each of the simulations. The material model parameters used were those determined previously for the best $R^{2}$ case.

In general, there was excellent correspondence between the numerical and constitutive model results (Fig. 11). There are some slight oscillations in the range of $\pm 0.1 \mathrm{MPa}$ for the numerical results for the $0.001-1 \mathrm{~s}^{-1}$ cases which were a result of the time scaling approach used

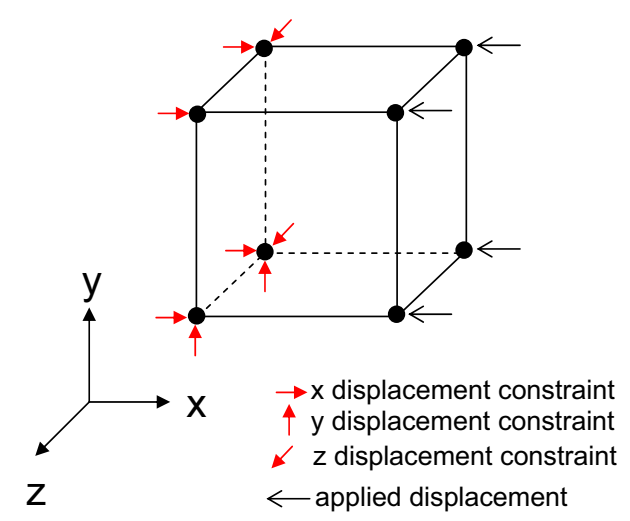

Fig. 10 Schematic of the simulations performed on the single solid element 
and the explicit nature of the numerical algorithm. To further highlight the accuracy of the numerical models, the $\mathrm{R}^{2}$ value for each curve was calculated between the experiment and numerical models (Table 3) and ranged from 0.9974 for the $1 \mathrm{~s}^{-1}$ case to 0.9999 for the $2700 \mathrm{~s}^{-1}$ with an average of 0.9983 for all cases demonstrating excellent agreement.

\section{Summary}

The objectives of this study were to: measure the mechanical properties of non-porous polychloroprene rubber across a range of strain rates, determine the parameters for a representative constitutive model and implement and validate the constitutive model for use in a large explicit finite element model. These developments were essential to support the second phase of this study investigating the mechanical response of porous polychloroprene rubber at the micro-scale. The constitutive model formulation developed in this study offers several advantages over currently available models. As noted by Ogden [8], the use of principal stretches highlights the isotropic nature of the elasticity of the material and through the use of invariants in the modifier term, the material maintains its objectivity and no further rotations, outside of those already required by the Ogden material model, need to be considered. It was shown that the model is capable of representing the mechanical behavior of rubber to very small compressive stretches (as low as 0.2 , or $80 \%$ compressive engineering strain). When implemented into finite element programs, the deformation gradient, $\mathbf{F}$, is often available whereas $\dot{\mathbf{F}}$ as required by the implementation in Eq. 8 requires further calculation

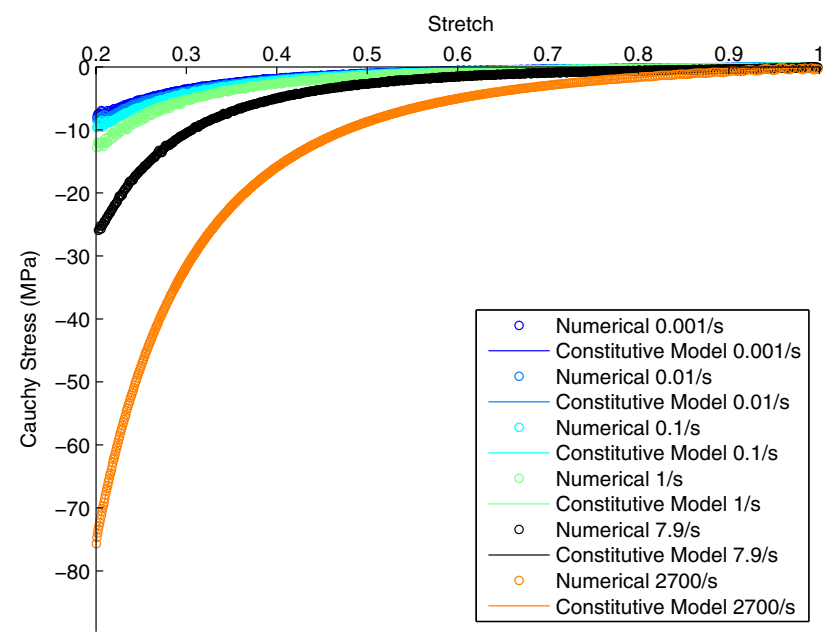

Fig. 11 Results of the single element numerical model compared to the constitutive model for the best $R^{2}$ case
Table 3 Individual $\mathrm{R}^{2}$ comparison of constitutive model to numerical model

\begin{tabular}{ll}
\hline Strain rate $\left(\mathrm{s}^{-1}\right)$ & $\mathrm{R}^{2}(-)$ \\
\hline 0.001 & 0.99758 \\
0.01 & 0.99661 \\
0.1 & 0.99816 \\
1 & 0.99741 \\
7.9 & 0.99982 \\
2700 & 0.99993 \\
Average & 0.99825 \\
\hline
\end{tabular}

[6] and additional memory storage (an additional nine real numbers), which was computationally prohibitive for the large finite element calculations undertaken in Part II of this study and addressing a limitation of existing models (e.g. Yang et al.). Additionally, Yang et al.'s assumption of only one coefficient for the Prony series was an arbitrary choice for ease of computation and the more general approach presented in this manuscript provided a more accurate representation of the properties for the polychloroprene. Rather than assuming the number of Prony series constants as was done in Yang et al., an iterative approach was taken to determine the number of coefficients required to accurately represent the material data. Limitations of the present method include fitting only to uniaxial test data and the direct use of a Prony series, rather than a physically representative model. However, since the goal was to implement the model in a numerical code, a Prony series approach was required and therefore was directly pursued.

Finally, the use of principal stretches in both the hyperelastic and viscoelastic stress contributions required the storage of three components compared to that of the standard implementation in finite element codes which requires the storage of six components (assuming symmetry) further reducing the memory requirement and providing a model that was self-consistent.

Material parameters were determined using a nonlinear optimization technique and the overall coefficient of determination or $R^{2}$ value was 0.9962 , indicating that the constitutive model was capable of representing the material response over a large range of strain rates and stretches. This model was implemented into an explicit finite element program and the results were validated using single element models $\left(R^{2}=0.9983\right)$ demonstrating the ability of the numerical implementation to represent larger deformations and a wide range of strain rates, an essential requirement for the second part of this study concerning micro-scale modeling of porous polychloroprene rubber.

Acknowledgments The authors gratefully thank Ushnish Basu at LSTC for his support and assistance in implementing the constitutive model, the Natural Sciences and Engineering Research Council of Canada (NSERC) for financial support. 


\section{Appendix 1: General Discretization of the Convolution Integral}

Recursive techniques can be used to solve the convolution integral of the type $F(t)=\int_{-\infty}^{t} k(t-\phi) \frac{d u}{d t}(\phi) d \phi$ using a numerical time marching approach [27, 28]. This can be explained through investigation of a generic convolution integral as that given by

$B(t)=\int_{-\infty}^{t} G(t-\phi) \frac{d A(\phi)}{d \phi} d \phi$

where, for this section only $B$ and $A$ can be stress and strain quantities respectively (written in a one dimensional form here) and $G$ is an independent function of time. It is usually assumed that the deformation starts at time zero resulting in

$B(t)=\int_{0}^{t} G(t-\phi) \frac{d A(\phi)}{d \phi} d \phi$

If a time marching technique is used and we take an increment in time $\Delta t$, Eq. 23 becomes

$B(t+\Delta t)=\int_{0}^{t+\Delta t} G(t+\Delta t-\phi) \frac{d A(\phi)}{d \phi} d \phi$

which can be split into the two intervals $[0, t]$ and $(t, t+\Delta t]$ resulting in

$$
\begin{aligned}
B(t+\Delta t) & =\int_{0}^{t} G(t+\Delta t-\phi) \frac{d A(\phi)}{d \phi} d \phi \\
& +\int_{t}^{t+\Delta t} G(t+\Delta t-\phi) \frac{d A(\phi)}{d \phi} d \phi
\end{aligned}
$$

The kernel function, $G(t+\Delta t-\phi)$, can be approximated by a Prony series, $\sum_{k=1}^{N_{k}} \gamma_{k} e^{-\beta_{k}(t+\Delta t-\phi)}$ where $N_{k}$ is the number of terms in the Prony series. The second term in Eq. 25 is then written as

$$
\begin{aligned}
& \int_{t}^{t+\Delta t} G(t+\Delta t-\phi) \frac{d A(\phi)}{d \phi} d \phi \\
& =\sum_{k=1}^{N_{k}} \gamma_{k} \int_{t}^{t+\Delta t} e^{-\beta_{k}(t+\Delta t-\phi)} \frac{d A(\phi)}{d \phi} d \phi
\end{aligned}
$$

where $\gamma_{k}$ and $\beta_{k}$ are constants. Using the exponential property $e^{c(a+b)}=e^{c a} e^{c b}$, Eq. 26 becomes

$$
\begin{aligned}
& \sum_{k=1}^{N_{k}} \gamma_{k} \int_{t}^{t+\Delta t} e^{-\beta_{k}(t+\Delta t-\phi)} \frac{d A(\phi)}{d \phi} d \phi \\
& =\sum_{k=1}^{N_{k}} \gamma_{k} \int_{t}^{t+\Delta t} e^{-\beta_{k}(t+\Delta t)} e^{\beta_{k} \phi} \frac{d A(\phi)}{d \phi} d \phi
\end{aligned}
$$

Applying the mean value theorem to Eq. 27 to extract the $A$ term from the integrand results in

$$
\begin{aligned}
& \sum_{k=1}^{N_{k}} \gamma_{k} \int_{t}^{t+\Delta t} e^{-\beta_{k}(t+\Delta t)} e^{\beta_{k} \phi} \frac{d A(\phi)}{d \phi} d \phi \\
& =\sum_{k=1}^{N_{k}} \gamma_{k} \frac{d A(\xi)}{d \phi} \int_{t}^{t+\Delta t} e^{-\beta_{k}(t+\Delta t)} e^{\beta_{k} \phi} d \phi
\end{aligned}
$$

where $\xi \in[t, t+\Delta t]$. The integral can now be evaluated to give

$\sum_{k=1}^{N_{k}} \gamma_{k} \frac{d A(\xi)}{d \phi} \int_{t}^{t+\Delta t} e^{-\beta_{k}(t+\Delta t)} e^{\beta_{k} \phi} d \phi$
$=\left.\sum_{k=1}^{N_{k}} \gamma_{k} \frac{d A(\xi)}{d \phi}\left[\frac{e^{-\beta_{k}(t+\Delta t-\phi)}}{\beta_{k}}\right]\right|_{t} ^{t+\Delta t}$

which simplifies to

$=\sum_{k=1}^{N_{k}} \frac{\gamma_{k}}{\beta_{k}} \frac{d A(\xi)}{d \phi}\left[1-e^{-\beta_{k} \Delta t}\right]$

If $d \phi$ is sufficiently small and equal to $\Delta t, d A / d \phi$ can be approximated linearly by

$\frac{d A}{d \phi}=\frac{A(t+\Delta t)-A(t)}{\Delta t}$

and finally Eq. 29 becomes

$$
\begin{array}{rl}
\int_{t}^{t+\Delta t} & G(t+\Delta t-\phi) \frac{d A(\phi)}{d \phi} d \phi \\
= & \sum_{k=1}^{N_{k}} \frac{\gamma_{k}}{\beta_{k}} \frac{A(t+\Delta t)-A(t)}{\Delta t}\left[1-e^{-\beta_{k} \Delta t}\right]
\end{array}
$$

which can be implemented into a time marching numerical approach.

Similarly, if we apply the Prony series approximation for the first term of Eq. 25 we get

$$
\int_{0}^{t} G(t+\Delta t-\phi) \frac{d A(\phi)}{d \phi} d \phi=\sum_{k=1}^{N_{k}} \int_{0}^{t} \gamma_{k} e^{-\beta_{k}(t+\Delta t-\phi)} \frac{d A(\phi)}{d \phi} d \phi
$$

separating the exponential terms results in

$=\sum_{k=1}^{N_{k}} \int_{0}^{t} \gamma_{k} e^{-\beta_{k}(t-\phi)} e^{-\beta_{k} \Delta t} \frac{d A(\phi)}{d \phi} d \phi$

which rearranged becomes

$=\sum_{k=1}^{N_{k}} e^{-\beta_{k} \Delta t} \int_{0}^{t} \gamma_{k} e^{-\beta_{k}(t-\phi)} \frac{d A(\phi)}{d \phi} d \phi$. If we substitute a Prony series, given by $\sum_{k=1}^{N_{k}} \gamma_{k} e^{-\beta_{k}(t-\phi)}$,
into Eq. 23 we get 


$$
\begin{aligned}
B(t) & =\int_{0}^{t} \sum_{k=1}^{N_{k}} \gamma_{k} e^{-\beta_{k}(t-\phi)} \frac{d A(\phi)}{d \phi} d \phi \\
& \equiv \sum_{k=1}^{N} H_{k}(t)
\end{aligned}
$$

where $H_{k}(t)$ is defined as a history variable for each $k$ and $H_{k}(0)=0$. Substituting this result into Eq. 33 results in

$$
\int_{0}^{t} G(t+\Delta t-\phi) \frac{d A(\phi)}{d \phi} d \phi=\sum_{k=1}^{N_{k}} e^{-\beta_{k} \Delta t} H_{k}(t) .
$$

Substituting Eqs. 32 and 33 into Eq. 25 gives the recursive formula

$$
\begin{aligned}
B(t+\Delta t)= & \sum_{k=1}^{N_{k}} e^{-\beta_{k} \Delta t} H_{k}(t) \\
& +\frac{\gamma_{k}}{\beta_{k}} \frac{A(t+\Delta t)-A(t)}{\Delta t}\left[1-e^{-\beta_{k} \Delta t}\right] .
\end{aligned}
$$

Using a time marching approach, one can then evaluate $B(t+\Delta t)$ using the values of $A$ at $t$ and $t+\Delta t$ and the history variable $H_{k}$ at $t$ along with the constants $\gamma_{k}$ and $\beta_{k}$ where $k=1$ to $N_{k}$.

There are different variations of the convolution in the literature which lead to different formulations. For instance

$\sigma_{\text {viscoelastic }}=\int_{0}^{t} g_{i j k l}(t-\phi) \frac{d \epsilon_{k l}(\phi)}{d \phi} d \phi$,

relates Cauchy stress to true (logarithmic) strain,

$S_{\text {viscoelastic }}=\int_{0}^{t} G_{i j k l}(t-\phi) \frac{d E(\phi)}{d \phi} d \phi$,

relates 2nd Piola-Kirchhoff stress to Green's strain,

$\sigma_{\text {viscoelastic }}=\int_{0}^{t} g(t-\phi) \frac{d \sigma[\epsilon(\phi)]}{d \phi} d \phi$,

as implemented in the modified quasi-linear form by Fung. Although $G_{i j k l}$ and $g_{i j k l}$ are 4 th order tensor quantities, they can be decomposed into scalar functions which act on the deviatoric and hydrostatic components.

\section{Appendix 2: General Stress Update Procedure}

The general solution procedure used a time marching technique as follows. At the current time increment (n) with time step $d t$ :

1. Given $F_{i j}$ solve for the principal stretches (eigenvalues) $\lambda_{i}=U_{i i}^{p}$ and the principal directions (eigenvectors).

2. Calculate the principal hyperelastic Kirchhoff stresses, $\tau_{i i}^{e}$, via Eq. 2 using $\lambda_{i}$ and material coefficients.
3. Calculate principal invariants of $\mathbf{U}$ so that

$$
\begin{aligned}
I_{1} & =\operatorname{tr}(\mathbf{U})=\lambda_{1}+\lambda_{2}+\lambda_{3} \\
I_{2} & =\frac{1}{2}\left[(\operatorname{tr} \mathbf{U})^{2}-\operatorname{tr}\left(\mathbf{U}^{2}\right)\right] \\
& =\left(\lambda_{1}+\lambda_{2}+\lambda_{3}\right)^{2}-\left(\lambda_{1}^{2}+\lambda_{2}^{2}+\lambda_{3}^{2}\right)
\end{aligned}
$$

4. Calculate viscoelastic modifier, (Eq. 13).

5. Use recursive techniques to solve convolution integral as follows (note that the history variables are set to zero at the start of the calculation):

(a) (a) Calculate the stretch rate

$$
\dot{\lambda}_{i}=\frac{\lambda_{i}^{n}-\lambda_{i}^{n-1}}{\Delta t} \text {. }
$$

(b) Calculate the increment for each Prony series coefficient and each direction (Eq. 16). These are the new history variables.

(c) Store the new history variables and new principal stretches for the next time step.

(d) Calculate the increment in viscoelastic stress for each direction (Eq. 17) by summing the new history variables calculated in step 5 b.

6. Sum the principal elastic and principal viscoelastic stresses to obtain the total principal stresses, i.e. $\tau_{i i}^{T}=$ $\tau_{i i}^{e}+\tau_{i i}^{v}$ (no summation on subscripts).

7. Calculate the dyadic product of the principal direction vectors, $n_{i} n_{j}$.

8. Calculate the Cauchy stress via $\sigma_{i j}=\tau_{i i} n_{i} n_{j} / J$.

Note that in the above procedure $\sigma \approx \tau$ since $J \approx 1$.

\section{References}

1. Meyers MA (1994) Dynamic behavior of material. Wiley, New York

2. Kolsky H (1949) An investigation of the mechanical properties of materials at high rates of loading. Proc Phys Soc B 62:676-700

3. Salisbury CP, Cronin DS (2009) Mechanical properties of ballistic gelatin at high deformation rates. Exp Mech 49(6):829-840

4. Dioh NN, Leevers PS, Williams JG (1993) Thickness effects in split Hopkinson pressure bar tests. Polymer 34:4230-4423

5. Yang LM, Shim VPW, Lim CT (2000) A visco-hyperelastic approach to modelling the constitutive behavior of rubber. Int $\mathrm{J}$ Impact Eng 24:545-560

6. Hallquist J (1998) LS-DYNA theoretical manual. LSTC, Livermore

7. Dassault Systemes (2008) ABAQUS theory manual version 6.8. Dassault Systemes, Providence

8. Ogden RW (1972) Large deformation isotropic elasticity-on the correlation of theory and experiment for incompressible rubber like solids. Proc R Soc Lond Ser A 326:565-584

9. Rivlin RS, Saunders DW (1951) Large elastic deformations of isotropic materials. VII. Experiments on the deformation of rubber. Philos Trans R Soc Lond Ser A 243(865):251-288 
10. Fung YC (1972) Stress-strain history relations of soft tissues in simple elongation. In: Biomechanics: its foundations and objectives. Prentice-Hall, Englewood Cliffs

11. Ouellet S, Cronin DS, Worswick MJ (2006) Compressive response of polymeric foams under quasi-static, medium and high strain rate conditions. Polym Test 256:731-743

12. Van Sligtenhorst C, Cronin DS, Brodland GW (2006) High strain rate compressive properties of bovine muscle tissue found using a split Hopkinson bar apparatus. J Biomech 39:1852-1858

13. Cronin DS, Williams KV, Salisbury CP (2011) Development and evaluation of a physical surrogate leg to predict landmine injury. J Mil Med 176(2):1408-1416

14. Salisbury CP (2001) Spectral analysis of wave propagation through a polymeric Hopkinson bar. MASc Thesis, University of Waterloo

15. Liu Q, Subhash G (2006) Characterization of viscoelastic properties of polymer bar using iterative deconvolution in the time domain. Mech Mater 38:1105-1117

16. Bacon C (1998) An experimental method for considering dispersion and attenuation in a viscoelastic Hopkinson bar. Exp Mech 38:242-249. doi:10.1007/BF02410385

17. Chen W, Zhang B, Forrestal MJ (1999) Split Hopkinson bar techniques for low impedance materials. Exp Mech 39:81-85. doi:10.1007/BF02331109
18. Crisfield MA (1991) Non-linear finite element analysis of solids and structures V1 and V2. Wiley, New York

19. Simo JC, Hughes TRJ (1998) Computational inelasticity. Springer, New York

20. Malvern LE (1969) Introduction to the mechanics of a continuous media. Prentice-Hall, Englewood Cliffs

21. Doman DA, Cronin DS, Salisbury CP (2006) Characterization of polyurethane rubber at high deformation rates. Exp Mech 46(3):367-376

22. Wilkinson L (1990) SYSTAT: the system for statistics. SYSTAT Inc., Evanston

23. Chen T (2000) Determining a prony series for a viscoelastic material from time varying strain data. NASA

24. MathWorks (2009) Matlab R2009b. MathWorks Inc., Massachusetts

25. Salisbury CP (2011) On the deformation mechanics of hyperelastic porous media. PhD Thesis, University of Waterloo

26. Doman DA (2004) Modeling of the high rate behavior of polyurethane rubber. MASc Thesis, University of Waterloo

27. Puso MA, Weiss JA (1998) Finite element implementation of anisotropic quasi-linear viscoelasticity using a discrete spectrum approximation. Trans ASME 120:62-79

28. Christensen RM (1980) A nonlinear theory of viscoelasticity of application to elastomers. J Appl Mech 47:762-768 